\title{
Zweikernige Au(II)-Verbindungen durch Disulfid-Addition an einen $\mathrm{Au}(\mathrm{I})$-Heterocyclus
}

H. Schmidbaur und J.R. Mandl

Anorganisch-chemisches Institut der Technischen Universität München

Die Oxidationsstufe + II ist bei Gold nur außerordentlich selten beobachtet worden [1]. $\mathrm{Zu}$ den weniger echten einkernigen $\mathrm{Au}^{2+}$-Verbindungen sind kürzlich Beispiele für Komplexe der zweikernigen Einheit $\mathrm{Au}_{2}^{4+}$ hinzugefügt worden [2]. Zur Darstellung wurde die transannulare oxydative Addition von Halogen $\left(\mathrm{Cl}_{2}\right.$, $\left.\mathrm{Br}_{2}, \mathrm{I}_{2}\right)$ an cyclische $\mathrm{Au}(\mathrm{I})-\mathrm{Komplexe}$ von Phosphor-Yliden benützt, die zum Aufbau einer starken $\mathrm{Au}$ - - Au-Bindung führt. Letztere entspricht der $\mathrm{Hg}-\mathrm{Hg}$-Bindung in den $\mathrm{Hg}(\mathrm{I})$-Verbindungen.

Jetzt konnte gezeigt werden, daß analoge Systeme auch durch oxydative Addition eines Disulfids erhältlich sind. Das farblose dimere Gold(I)-diäthyl-phosphonium-bismethylid 1 wird von Thiuramdisulfid schon bei Raumtemperatur in Methylenchlorid mit hohen Ausbeuten in ein rot gefärbtes Addukt 2 umgewandelt, das auf Zusatz von Pentan gut kristallisiert (Schmp. $120^{\circ} \mathrm{C}$ ):

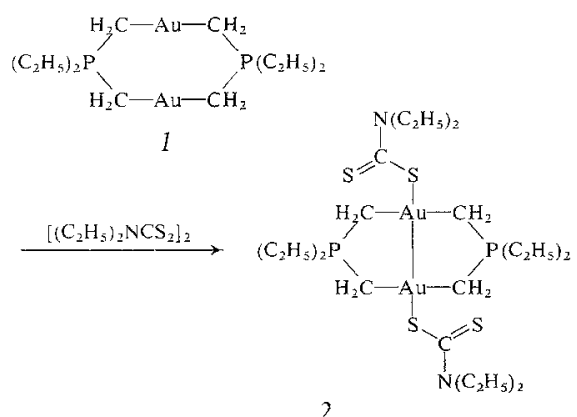

Der Strukturvorschlag $2^{\star}$ stuitzt sich auf folgende analytische und spektroskopische Daten:

Elementaranalyse $\quad \mathrm{C}_{22} \mathrm{H}_{48} \mathrm{Au}_{2} \mathrm{~N}_{2} \mathrm{P}_{2} \mathrm{~S}_{4}$ (924,75: ber. C28,57, H5,23, Au 42,60; gef. C28,55, H 5,22, Au 42,70. - ${ }^{1} H-N M R$

\footnotetext{
* Nomenklatur: $\mathrm{Au}, \mathrm{Au}^{\prime}$-Bis-dithiocarbamato-digold(II)- $\mu, \mu^{\prime}$-bis-(diäthylphosphonium-bis-methylid)
}

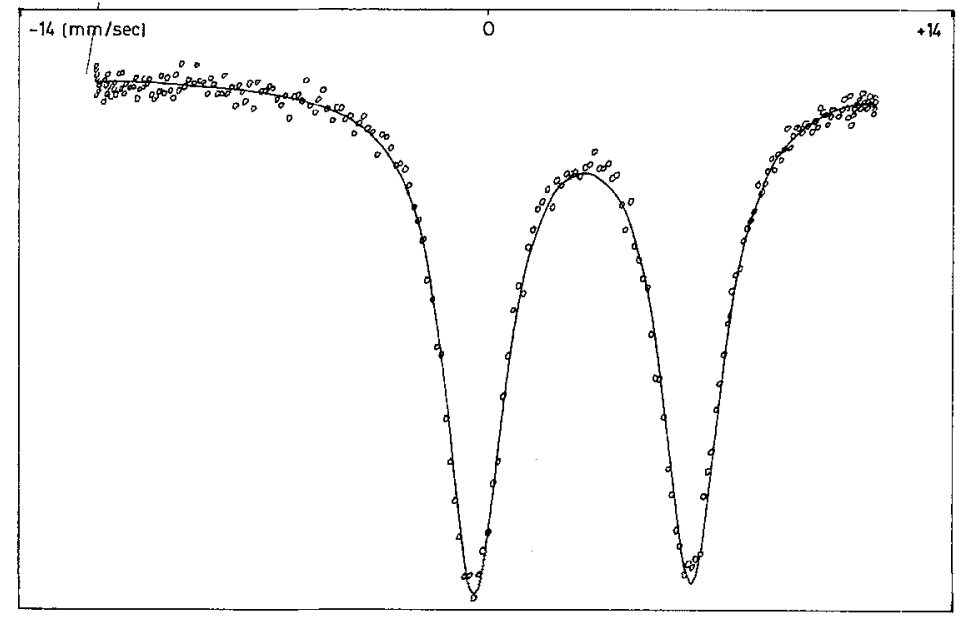

Fig. 1. ${ }^{197} \mathrm{Au}-\mathrm{MößBbauer-Spektrum}$ von 2

$\left(\mathrm{CDCl}_{3}, \mathrm{TMS}\right): \delta \mathrm{CH}_{2} \mathrm{Au} 1,70 \mathrm{ppm}, \mathrm{d}, 4 \mathrm{H}$, $J$ (HCP) $10,5 \mathrm{~Hz} ;\left\{{ }^{31} \mathrm{P}\right\}$ : s. $\delta \mathrm{CH}_{2} \mathrm{P} 2,20, \mathrm{dq}$, $4 \mathrm{H}, J(\mathrm{HCP}) 11,5, J(\mathrm{HCCH}) 7 ; \delta \mathrm{CH}_{3} 1,60$, m, $12 \mathrm{H}$. - ${ }^{31} \mathrm{P}-\mathrm{NMR}\left(\mathrm{CH}_{2} \mathrm{Cl}_{2}, \mathrm{H}_{3} \mathrm{PO}_{4}\right)$ : $\delta \mathrm{P} 32,1$ ppm. - ${ }^{197} A u$-Mößbauer-Spektrum (4K): IS 2,90 $\mathrm{mm}^{-1}$; QS $6,50 \mathrm{~mm}^{-1}$. 2 ist diamagnetisch. ESCA-Spektrum: Au $(4 f)^{7} / 284,2 \mathrm{eV}$.

Diese Ergebnisse bestätigen, daß die kristallographisch erwiesene zentrosymmetrische Struktur [3] des Ausgangsmaterials 1 bei der oxydativen Addition erhalten bleibt. Insbesondere folgt aus dem Mößbauer(und dem Röntgenphotoelektronen-) Spektrum (Fig. 1) die Äquivalenz der beiden Goldatome und ihre Oxydationsstufe + II, denn die Werte für eine analoge Chlorverbindung [4], deren Struktur ebenfalls röntgenographisch erwiesen ist [5], sind jeweils nur wenig verschieden: IS 2,67 $\mathrm{mm}^{-1}$; QS $6,71 \mathrm{~mm}^{-1}$. Analoge Gold(I)- und Gold(III)-Komplexe zeigen dagegen stark abweichende Parameter. Der Diamagnetismus des Komplexes resultiert zwangsläufig aus der Spinabsättigung der beiden $\mathrm{d}^{9}$-konfigurierten Metallatome in der transannularen $\mathrm{Au}-\mathrm{Au}$-Bindung. Damit ist nachgewiesen, daß die Einheit $\mathrm{Au}_{2}^{4+}$ bei Gegenwart chelatisierender Gruppen auch durch Schwefel-Liganden stabilisierbar ist.

Wir danken der Deutschen Forschungsgemeinschaft und dem Fonds der Chemischen Industrie für die Unterstützung dieser Untersuchungen, Doz. Dr. F.E. Wagner, Physikdepartment (E 15) der TU München, für die Aufnahme der Mößbauer-Spektren, Prof. G.P. van der Kelen und Dr. D.F. van der Vondel, Univ. Gent, für das ESCA-Spektrum.

Eingegangen am 5. Oktober 1976

1. Bergendahl, T.J.: J. Chem. Educ. 52, 731 (1975)

2. Schmidbaur, H., Franke, R.: Inorg. Chim. Acta 13, 84 (1975)

3. Schmidbaur, H.,etal.: Chem. Ber. (im Druck)

4. Schmidbaur, H., et al.: Chem. Commun. 1976, 170

5. Schmidbaur, H., et al.: Chem. Ber. 109, 466 (1976)

\section{Zur Wärmeempfindlichkeit von oxischen und hypoxischen Zellen in einem Tumor}

F. Dietzel und H.J. Weber

Zentrum für Radiologie, Abteilung Nuklearmedizin, Universität Gießen

W. Porschen und L.E. Feinendegen

Institut für Medizin, Kernforschungsanlage Jülich $\mathrm{GmbH}$

Im soliden Tumor wird infolge der raschen und unkoordinierten Zellteilung an vielen Stellen die Diffusionsstrecke vom Versor- gungsgefäß zu den Tumorzellen so groß, $\mathrm{daB}$ diese Zellen zwangsläufig in eine Sauerstoff-Unterversorgung geraten. Hyp- oxische Tumorzellen sind jedoch weit weniger empfindlich gegenüber locker ionisierender Strahlung als gut sauerstoffversorgte.

Es gibt Untersuchungen, welche größere Wärmesensibilität von Tumorzellen in Sauerstoff-Unterversorgung vermuten lassen [1]. Die meisten dieser Hinweise resultieren jedoch aus Untersuchungen in Zellkulturen, da Zellkulturen leicht unter definierten Hypoxiebedingungen bestrahlt werden können. Es fehlt bisher der Beweis 